\title{
EVALUACIÓN DE LA CREDIBILIDAD DE LA PRUEBA TESTIFICAL EN VÍCTIMAS CON DISCAPACIDAD INTELECTUAL
}

\section{Credibility assessment of testimonies provided by victims with intellectual disabilities}

Antonio L. MAnZanero

Universidad Complutense de Madrid. Facultad de Psicología. Dpto. Psicología Básica I. Campus de Somosaguas. 28223 Madrid

antonio.manzanero@psi.ucm.es

Rocío VALLeT

Universidad Complutense de Madrid

Marina Nieto-MárqueZ

Universidad Complutense de Madrid

Susana BARÓN

Universidad Complutense de Madrid

M. ${ }^{\text {a Teresa SCOTT }}$

Universidad del Desarrollo. Chile

Recepción: 4 de julio de 2016

Fecha de aceptación definitiva: 31 de enero de 2017

Resumen: Uno de los principales obstáculos en el acceso a la justicia de las víctimas con discapacidad procede de los estereotipos referidos a su capacidad para declarar en un procedimiento policial y judicial, tendiendo unos a considerarlos menos creíbles que al resto de víctimas y otros más creíbles por su supuesta incapacidad para elaborar mentiras complejas. El presente trabajo revisa tres de los estudios recientemente realizados por el grupo UCM de Psicología del Testimonio con el objetivo de analizar el papel de la experiencia y la intuición en la evaluación de la credibilidad en personas con discapacidad intelectual (DI), y comprobar si los procedimientos de análisis de credibilidad tales como el basado en el Modelo de Control de la Realidad [Reality Monitoring, RM] y el de Evaluación de la Validez de las Declaraciones [Statement Validity Assessment, SVA] serían válidos para discriminar entre relatos verdaderos y falsos en estos colectivos. De 
los resultados de dichos estudios se deduce que la experiencia no parece ser suficiente para discriminar entre las víctimas reales y simuladas, pero tampoco parece resultar suficiente analizar las características de los relatos como único indicador. Se propone como alternativa el procedimiento general HELPT para la evaluación de la credibilidad en personas con DI.

Palabras clave: credibilidad; discapacidad intelectual; criterios de contenido; psicología del testimonio; juicios intuitivos; jurados, HELPT.

AвsTRAct: One of the main obstacles in the way of access to justice for the victims with intellectual disability comes from the stereotypes referred to their ability to produce a statement at police legal procedures, with the consequence that some consider their statements less reliable than the rest of the victims, and others considerate their statements more reliable given their inability to create complex lies. This article reviews three of the most recent studies done by the UCM group of Psychology of Testimony, with the objective of analyzing the role of experience and intuition in the evaluation of credibility in people with intellectual disability (ID), and also it aims to prove whether the credibility analysis procedures such as Reality Monitoring (RM) and Statement Validity Assessment (SVA) would be valid procedures to discriminate between real and false statements within these collectives. From the results of these studies, it can be deducted that experience may not seem to be enough in order to discriminate between real and simulated victims, but analyzing the characteristics of the statements as the only indicator doesn't seem to be enough either. As an alternative, the general procedure HELPT is proposed for the evaluation of credibility of people with ID.

KEY WORDS: credibility assessment; intellectual disability; content criteria; eyewitness testimony; intuitive judgments; jurors; HELPT.

\footnotetext{
S
} egún fuentes de la Policía Judicial de la Guardia Civil (González, Cendra y Manzanero, 2013), del 2008 al 2010 se interpusieron en España, en su competencia, 10.045 denuncias en las que estaba implicada una persona con discapacidad intelectual (DI) como víctima, agresor o testigo. Siendo alto el número de casos, sin embargo, las personas con DI tienden a ser excluidas por los sistemas judiciales debido a que tienden a ser menos creíbles o menos capaces de recordar que otros tipos de víctimas (Collins y Henry, 2016; Bettenay, Ridley, Henry y Crane, 2014; Brown y Lewis, 2013; Henry, Ridley, Perry y Crane, 2011; Nathanson y Platt, 2005; Peled, Iarocci y Connolly, 2004; Stobbs y Kebbell, 2003; Ternes y Yuille, 2008).

Sin embargo, se ha propuesto que mentir sería cognitivamente más complejo que decir la verdad (Vrij, Fisher, Mann y Leal, 2006; Massip y Herrero, 2015), por lo que implicaría una mayor demanda de recursos cognitivos (Vrij y Heaven, 1999). En este sentido, algunos de los tópicos existentes sobre la capacidad de las personas con DI llevarían a considerar que no serían capaces de elaborar mentiras complejas, por lo que deberían ser más creíbles (Bottoms, Nysse-Carris, Harris y Tyda, 2003).

Pese a que ambos tópicos se contradicen, es probable que influyan de forma simultánea en la capacidad para discriminar verdad y mentira en personas con DI. En 
cualquier caso, la falta de procedimientos adaptados a sus necesidades y el desconocimiento de las circunstancias específicas de la persona a evaluar podrían llevar a graves errores en la apreciación de sus testimonios.

\section{El papel de la experiencia y la intuición en la evaluación de la credibilidad en personas con discapacidad}

En general, podríamos decir que la capacidad que tenemos para evaluar la credibilidad de una declaración emitida por una persona con un desarrollo típico es relativamente escasa, tendiendo a estar próxima al azar. La mayoría de los estudios (Aamodt y Custer, 2006; Akehurst, Bull, Vrij y Köhnken, 2004; Bond, 2007; Bond y DePaulo, 2006; Mann y Vrij, 2006; Mann, Vrij y Bull, 2004; Manzanero y Diges, 1994) arrojan porcentajes de éxito al discriminar entre relatos reales y falsos cercanos al 50\%, con una desviación aproximada de $\pm 10 \%$. Este porcentaje de aciertos sería similar para distintos grupos dependiendo de su experiencia (policía, jueces, estudiantes, otros). La única diferencia que habitualmente se ha encontrado (Bond y DePaulo, 2006; Meissner y Kassin, 2002) es que los sujetos sin formación alguna en análisis de la credibilidad en general tienden a la veracidad, esto es, a creerse los relatos, mientras que los jueces y policías tienden más a la mendacidad, a pensar que los relatos son falsos.

No obstante, algunos estudios (Ekman, O’Sullivan y Frank, 1999; Ericsson y Smith, 1991) inicialmente señalaron que los expertos en este tipo de tareas, como son los psicólogos forenses, abogados o policías, tendrían mayor capacidad para discriminar los testimonios falsos de los verdaderos que los sujetos sin experiencia. Sin embargo, la mayoría de los estudios más recientemente hacen referencia a que no existen diferencias significativas en la capacidad para discriminar la verdad y la mentira entre evaluadores expertos y no expertos (Alonso, Masip y Garrido, 2009; Bond y DePaulo, 2006; Garrido, Masip y Herrero, 2004; Goodman, Batterman-Faunce, Schaaf y Kenney, 2002).

No obstante, estos datos podrían ser diferentes cuando de lo que se trata es de evaluar a una persona con DI. En una investigación (Manzanero, Quintana y Contreras, 2015) cuyo objetivo principal era analizar la capacidad intuitiva para discriminar entre declaraciones reales y falsas emitidas por personas con DI ligera y moderada (CI medio $=60,0$; rango $=50-80$ ), se compararon las evaluaciones emitidas por colectivos con distintos grados de experiencia en la obtención y la evaluación de declaraciones.

En el experimento participaron un grupo de evaluadores sin experiencia formado por estudiantes de Psicología y dos grupos de agentes de Policía Judicial con diferente grado de experiencia, "veteranos" con más de 10 años de servicio y "novatos" con menos de 10 años. Ninguno de los participantes tenía formación específica sobre discapacidad intelectual.

Los resultados de los análisis de credibilidad realizados por los estudiantes y los agentes de Policía Judicial mostraron que eran capaces de discriminar entre declaraciones reales y falsas por encima del azar, con criterios de respuesta neutros y un porcentaje de clasificaciones correctas del 65,63 y 62,24 por ciento respectivamente. 
La Tabla 1 muestra la frecuencia y porcentaje de aciertos (A), rechazos correctos (RC), Falsas Alarmas (FA) y Omisiones (O) obtenidos por cada grupo. Los resultados indicaron que el rendimiento de los agentes de la Policía Judicial de la Guardia Civil no es estadísticamente diferente de la obtenida por el grupo de control de los estudiantes.

\begin{tabular}{|l|l|c|c|}
\hline \multicolumn{4}{|c|}{$\begin{array}{c}\text { TABLA 1. Frecuencias y porcentaje de aciertos (A), rechazos correctos (RC), } \\
\text { falsas alarmas (FA) y Omisiones (O) obtenidos por cada grupo } \\
\text { (Manzanero, Quintana y Contreras, 2015) }\end{array}$} \\
\hline \multirow{2}{*}{ Estudiantes } & & \multicolumn{2}{|c|}{ ORIGEN DE LA DECLARACIÓN } \\
\cline { 2 - 4 } & Evaluación & Simulada & Real \\
\hline \multirow{2}{*}{ Pentira } & Verdad & RC: $308(66.52 \%)$ & O: $176(37.21 \%)$ \\
\hline \multirow{2}{*}{ Policías } & & $463(100 \%)$ & A: $297(62.79 \%)$ \\
\hline & Mentira & RC: $268(58.64 \%)$ & O: $130(33.50 \%)$ \\
\cline { 2 - 4 } & Verdad & FA: $189(41.35 \%)$ & A: $258(66.49 \%)$ \\
\hline
\end{tabular}

Los resultados obtenidos en función de los años de experiencia del grupo de policías mostraron que los agentes que tienen mayor antigüedad en la especialidad de Policía Judicial no muestran una mayor precisión en sus juicios.

Así pues, se pudo concluir que la experiencia en tareas relacionadas con la obtención y evaluación de declaraciones no resultaba relevante al evaluar intuitivamente las declaraciones de personas con DI, siendo que los porcentajes de aciertos y errores no resultaron estadísticamente diferentes, superando los errores en todos los casos el 30 por ciento. Estos datos apoyarían los estudios que ponen de relieve la ausencia de diferencias entre los evaluadores con y sin experiencia en discriminar entre declaraciones verdaderas y falsas emitidas por personas con un desarrollo intelectual típico (Bond y DePaulo, 2006; Goodman et al., 2002; Vrij y Graham, 1997).

La experiencia no parece ser suficiente para discriminar intuitivamente entre las víctimas reales y simuladas por lo que, como muestran algunos estudios, se deberían potenciar técnicas específicas que ayuden a realizar esta tarea con una menor tasa de error (Clarke, Prescott y Milne, 2013; Brown, Lewis, Lamb y Stephens, 2012; Carlin, Toglia, Belmonte y DiMeglio, 2012; Faccini y Saide, 2011; Kebbell, Hatton y Johnson, 2004).

\section{Modelo de Control de la Realidad (RM)}

En el marco de la psicología del testimonio se han propuesto diferentes procedimientos de evaluación de la credibilidad mediante el análisis del contenido de las 
declaraciones. Uno de estos procedimientos se basa en el modelo de Control de la Realidad (Reality Monitoring-RM) (Johnson y Raye, 1981; Johnson, Hashtroudi y Lindsay, 1993), que propone que las declaraciones que se basan en memorias de sucesos reales son diferentes en calidad de las declaraciones que no se basan en la experiencia, o de las que son meros productos de la fantasía, y por lo tanto podría ser útil en la evaluación de credibilidad (Manzanero, 2001; McNally, Clancy, Barrett y Parker, 2005; Otgaar, Candel, Memon y Almerigogna, 2010).

En una primera aproximación, Johnson y Raye (1981) propusieron la existencia de cuatro tipos de atributos esenciales mediante los que podríamos diferenciar entre las dos clases de información almacenadas en la memoria. Las memorias de origen perceptivo poseerían más atributos contextuales y sensoriales, y más detalles semánticos; mientras que los recuerdos autogenerados contendrían más información sobre operaciones cognitivas. A partir de posteriores investigaciones, el listado de atributos diferenciales ha ido aumentando (ver Tabla 2), al tiempo que los datos mostraban que la presencia de estos rasgos distintivos depende de la influencia de multitud de factores.

\section{TABla 2. Dimensiones de las descripciones de memoria que podrían ser relevantes} para discriminar su origen (Manzanero, 2010)

\begin{tabular}{|l|}
\hline Información sensorial \\
\hline Detalles sobre el contexto espacial y temporal del suceso (interno) \\
\hline Detalles sobre el contexto ambiental (externo) \\
\hline Alusiones a procesos cognitivos \\
\hline Expresiones dubitativas \\
\hline Información irrelevante o superflua \\
\hline Explicaciones \\
\hline Autorreferencias \\
\hline Exageraciones \\
\hline Implicación personal o perspectiva de recuperación \\
\hline Juicios y comentarios personales \\
\hline Muletillas \\
\hline Pausas \\
\hline Correcciones espontáneas \\
\hline Cambios de orden \\
\hline Longitud del relato \\
\hline
\end{tabular}

En cualquier caso, la presencia de rasgos característicos en las declaraciones reales en comparación con declaraciones sobre hechos imaginados o sugeridos dependería de la activación (Diges, Rubio y Rodríguez, 1992), los conocimientos previos (Diges, 1995), la modalidad perceptiva (Henkel, Franklin y Johnson, 2000), la preparación (Manzanero y Diges, 1995), el paso del tiempo (Manzanero, 2006; Manzanero, ElAstal y Aróztegui, 2009), la edad (Comblain, D’Argembeau y Van der Linden, 2005), la realización de preguntas y la recuperación múltiple (Manzanero, 1994; Strömwall, 
Bengtsson, Leander y Granhag, 2004) o factores contextuales (Campos y AlonsoQuecuty, 1998), además del tipo de diseño utilizado en las investigaciones realizadas sobre este tópico (Bensi, Gambetti, Nori y Giusberti, 2009).

Por otro lado, existe una gran variabilidad en el origen de los recuerdos. Así, las características diferenciales no son iguales para la fantasía, la mentira, los sueños o la información postsuceso. Pero incluso para cada modalidad hay diferentes grados de distancia con la información real. Por ejemplo, no es lo mismo inventarse un suceso completo que cambiar un detalle de un suceso real, por muy relevante que éste sea (Manzanero, 2009). Los relatos falsos nunca son inventados en su totalidad, sino que proceden en parte de información perceptiva de distintos orígenes, reelaborada para dar lugar a un relato nuevo.

De igual modo, las características de los relatos podrían variar en función de la capacidad del sujeto para generar un relato plausible. En este sentido, el grado y tipo de discapacidad intelectual podría ser un factor a tener en cuenta. Se hacía, por tanto, necesario conocer en qué medida los relatos emitidos por personas con DI presentaban los rasgos característicos en función de su origen según el modelo RM (Manzanero, Alemany, Recio, Vallet y Aróztegui, 2015). Los resultados alcanzados muestran que los relatos emitidos por víctimas reales o simuladas con DI moderada y leve solo diferían en cantidad de detalles y longitud. La falta de efectos en la mayoría de los criterios se debería a una enorme variabilidad, así como a la presencia de efecto suelo en algunos casos, puesto que los relatos resultan en general muy poco ricos fenomenológicamente. A esto hay que sumarle que estos dos rasgos se ven afectados por gran variedad de factores, como por ejemplo el tipo de hecho descrito, el paso del tiempo o las capacidades del testigo. La mayoría de las personas con DI tienen dificultad para contar con riqueza de detalles un suceso vivido, incluso tienden con mayor probabilidad que la población sin DI a no incluir los detalles relevantes del mismo (Perlman, Ericson, Esses y Isaacs, 1994). Así mismo, muchas personas con DI tienen muchas dificultades para incardinar los eventos en un tiempo y un espacio (Bailey et al., 2004; Landau y Zukowsky, 2003).

Estos datos permiten concluir que el modelo de RM no permite discriminar entre relatos aportados por víctimas reales y simuladas con DI.

\section{Análisis de la credibilidad de los relatos de personas con DI mediante CBCA}

Desde un punto de vista aplicado, la psicología forense ha propuesto otros procedimientos de evaluación de la credibilidad mediante el análisis del contenido de las declaraciones. Uno de estos procedimientos es la técnica Statement Validity Analysis o SVA (Steller y Köhnken, 1989) que permite la valoración de la credibilidad de las declaraciones emitidas por menores víctimas de abusos sexuales. Su utilidad sería poder aportar información relevante para la toma de decisión acerca de la hipótesis de que el relato emitido por la supuesta víctima tuviera su origen en una mentira deliberada (Köhnken, Manzanero y Scott, 2015).

El procedimiento SVA incluye un listado de criterios, el CBCA (criteria-based content analysis), que tiene en cuenta 19 criterios de contenido enmarcados en cinco 
categorías: características generales, contenidos específicos de la declaración, peculiaridades del contenido, contenidos relacionados con la motivación y elementos específicos de la agresión (Tabla 3).

\begin{tabular}{|c|c|}
\hline \multicolumn{2}{|r|}{$\begin{array}{l}\text { Tabla 3. Criterios de contenido para la evaluación de la credibilidad } \\
\text { de los relatos (Steller y Köhnken, 1989) }\end{array}$} \\
\hline \multicolumn{2}{|r|}{ Características generales } \\
\hline 1. & Estructura lógica \\
\hline 2. & Producción no estructurada \\
\hline 3. & Cantidad de detalles \\
\hline \multicolumn{2}{|c|}{ Contenidos especificos } \\
\hline 4. & Incardinación en contexto \\
\hline 5. & Descripción de interacciones \\
\hline 6. & Reproducción de conversaciones \\
\hline 7. & Complicaciones inesperadas \\
\hline \multicolumn{2}{|c|}{ Peculiaridades de contenido } \\
\hline 8. & Detalles inusuales \\
\hline 9. & Detalles superfluos \\
\hline 10. & Detalles exactos mal interpretados \\
\hline 11. & Asociaciones externas relacionadas \\
\hline 12. & Estado mental subjetivo de la víctima \\
\hline 13. & Atribuciones al estado mental del agresor \\
\hline \multicolumn{2}{|c|}{ Contenido relacionado con motivación } \\
\hline 14. & Correcciones espontáneas \\
\hline 15. & Admisión de falta de memoria \\
\hline 16. & Dudas sobre el propio testimonio \\
\hline 17. & Autodesaprobación \\
\hline 18. & Perdón del acusado \\
\hline \multicolumn{2}{|c|}{ Elementos especificos de la agresión } \\
\hline 19. & Detalles característicos del tipo de agresión \\
\hline
\end{tabular}

El supuesto básico del CBCA es que las declaraciones que se basan en memorias de sucesos reales son diferentes en calidad de las declaraciones que no se basan en la experiencia o de las que son meros productos de la fantasía tal y como ya había puesto de manifiesto Undeutsch (1982). Según la propuesta original (Steller y Köhnken, 1989), cada criterio de contenido es un indicador de la credibilidad de una declaración, aunque su ausencia no significa necesariamente que la declaración sea falsa. No obstante, este supuesto se ha revelado erróneo, ya que tanto la presencia como la ausencia podrían indicar credibilidad y no todos los criterios son relevantes a la hora de discriminar (Bekerian y Dennett, 1992; Porter y Yuille, 1996; Sporer y Sharman, 2006; Vrij, 2005; Vrij, Akehurst, Soukara y Bull, 2004). Por esta razón, la presencia de 
estos criterios no se puede interpretar en ausencia de un análisis previo de los factores que podrían afectarles (Köhnken et al., 2015).

En general, podemos afirmar que la inconsistencia de los atributos diferenciales caracteriza los estudios que han tratado de encontrar diferencias entre relatos verdaderos y falsos (producto de la mentira y la imaginación). Masip, Sporer, Garrido y Herrero (2005) revisaron algunos de los trabajos que durante los noventa y primeros años de la siguiente década analizaron las diferencias entre relatos reales y falsos. La conclusión a la que llegan es que no todos los criterios aparecen relacionados con los relatos verdaderos, y argumentan que se debería a la falta de una teoría que permita explicar cuándo, cómo y por qué aparecen estos criterios. En esta misma dirección, Sporer (1997) afirmaba que las supuestas diferencias entre declaraciones reales y falsas son una mera hipótesis de trabajo sobre la que no se puede especificar por qué se dan esas diferencias, cuáles son los procesos psicológicos que las sustentan ni las condiciones que hacen que puedan aparecer o no en una declaración.

No obstante, esta técnica se ha utilizado durante décadas no solo para menores supuestas víctimas de abusos sexual, sino que desde sus inicios también se aplicó a personas con DI, ya que cumplían la mayoría de los requisitos necesarios para su utilización:

- Los hechos deben tener connotaciones sexuales.

- La víctima no debe tener experiencia previa o conocimientos sexuales. Razón por la cual en general no es de aplicación a adolescentes.

- El suceso debe ser lo suficientemente complejo como para que el relato contenga información suficiente para ser analizada e ir más allá de la capacidad de invención de la posible víctima.

- Relato espontáneo.

- Una víctima con capacidad suficiente como para aportar un relato lo suficientemente detallado de forma espontánea. Téngase en cuenta que en los menores hasta los 6 años no se habrían completado suficientemente el desarrollo de capacidades cognitivas y metacognitivas, y en las personas con DI puede haber alteraciones o déficits significativos.

En cualquier caso, se hace necesario analizar en qué medida los criterios de CBCA pueden ser útiles para un análisis de credibilidad en personas con DI. Por esta razón, en una investigación (Manzanero, Vallet, Aróztegui y Scott, en revisión) se procedió a analizar los relatos de las víctimas reales o simuladas utilizados en las investigaciones anteriores (Manzanero, Quintana et al., 2015; Manzanero, Alemany et al., 2015) mediante los criterios de CBCA. El análisis de los datos mostró que resultan significativos únicamente los criterios de cantidad de detalles, incardinación en contexto y reproducción de conversaciones; no habiendo efectos significativos sobre el resto de los criterios.

La pregunta que surge es si los criterios que nos pueden servir para discriminar entre relatos verdaderos o falsos son la cantidad de detalles, la incardinación en contexto y la reproducción de conversaciones, y siendo el primero además especialmente relevante para valorar un testimonio como real, ¿qué sucede con todas aquellas personas con un 
vocabulario reducido, con déficit en la memoria semántica y autobiográfica (que no les permita reproducir adecuadamente las conversaciones) o con dificultad para incardinar los eventos en un contexto dado? Corremos el riesgo de que las personas con DI que presenten dichos déficits puedan sufrir un juicio intuitivo de credibilidad erróneo, con la consecuente revictimización que ello supondría.

Por esta razón, se hace imprescindible conocer todos los factores que podrían afectar a las características de los relatos, si no se hiciera así se correría el riesgo de alcanzar conclusiones erróneas.

\section{Alternativas al uso de criterios}

Ya desde las primeras propuestas en los años 70 y 80 del pasado siglo se propusieron procedimientos complejos que iban más allá de observar la mera presencia o ausencia de una serie de criterios (ver, por ejemplo, Undeutsch, 1984; Steller y Köhnken, 1989). En este sentido el CBCA es sólo una herramienta dentro de un protocolo más general que considera otros factores como las capacidades de la víctima, las condiciones en que se han obtenido las declaraciones, otras cuestiones de la investigación

La investigación en Psicología del Testimonio durante las últimas décadas nos ha ido mostrando algunos de los factores de contaminación de la prueba testifical más relevantes que deberían incorporarse a estos procedimientos.

El método Holístico de Evaluación de La Prueba Testifical (HELPT; Manzanero y González, 2013, 2015) contempla la evaluación de todos los aspectos relevantes en la obtención y valoración de un testimonio, y es especialmente indicado para víctimas vulnerables (menores, personas con DI, personas con enfermedad mental o ancianos). El principal objetivo del procedimiento HELPT es obtener toda la información posible de los testigos y víctimas de un hecho delictivo, con la mínima interferencia, y valorar su credibilidad.

Para la evaluación de la credibilidad de la declaración, el HELPT propone: 1) analizar los factores de influencia a partir de los datos del expediente (Scott y Manzanero, 2015); 2) evaluar la capacidad para testificar y de los conocimientos previos, para adaptar los procedimientos a las características de la víctima y tener elementos objetivos de análisis de la información aportada por la víctima (Contreras, Silva y Manzanero, 2015); 3) planteamiento de hipótesis, en función del caso concreto, para posteriormente diseñar el procedimiento de validación más adecuado (Tabla 4); 4) obtener la declaración mediante procedimientos adaptados a la víctima minimizando la posibilidad de contaminación de la declaración; y 5) análisis/evaluación de la declaración.

Para la evaluación de las pruebas de identificación, se propone: 1) evaluar la capacidad para identificar; 2) analizar los factores del suceso y del testigo que pueden afectar a la exactitud de las identificaciones; 3) obtención de la descripción; 4) obtención del retrato-robot (si fuese necesario); 5) diseño y realización de la prueba de identificación; 6) análisis/evaluación de la identificación; 7) evaluación de la validez de la identificación; y 8) posibles causas de falsas identificaciones. 


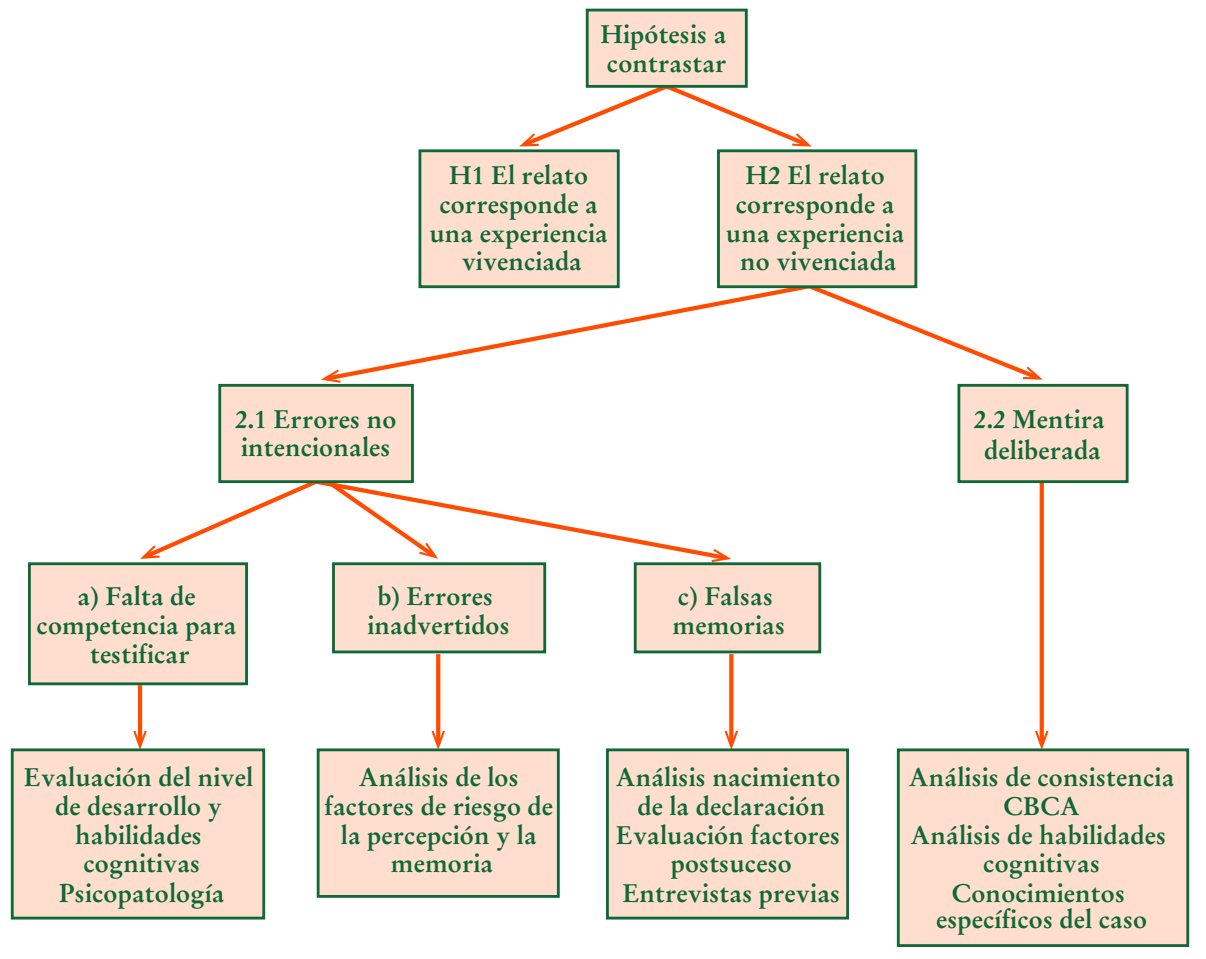

Como puede observarse, resulta de especial interés en este procedimiento la evaluación de las capacidades para testificar de la víctima, para lo que se han diseñado herramientas específicas como el CAPALIST (Contreras et al., 2015), procedimientos de entrevista convenientemente adaptados (González, Muñoz, Sotoca y Manzanero, 2013), y considerar factores y métodos específicos para la obtención y evaluación de la prueba de identificación (Manzanero, 2010).

\section{Referencias bibliográficas}

Aamodt, M. G. y Custer, H. (2006). Who can best catch a liar? A meta-analysis of individual differences in detecting deception. Forensic Examiner, 15 (1), 6-11.

Akehurst, L., Bull, R., VRij, A. y Köhnken, G. (2004). The effects of training professional groups and lay persons to use criteria-based content analysis to detect deception. Applied Cognitive Psychology, 18 (7), 877-891. doi:10.1002/acp.1057.

Alonso, H., Masip, J. y Garrido, E. (2009). La capacidad de los policías para detectar mentiras. Revista de Derecho Penal y Criminología, 2, 159-196. 
Bailey, D. B., Roberts, J. E., Hooper, S. R., Hatton, D. D., Mirrett, P. L., Roberts, J. E. y SchaAf, J. M. (2004). Research on fragile X syndrome and autism: implications for the study of genes, environments and developmental language disorders. En M. L. RicE y S. F. WARREN (Eds.), Developmental language disorders: From phenotypes to etiologies (pp. 121150). Mahwah, NJ: Erlbaum.

Bekerian, D. A. y DennetT, J. L. (1992). The truth in content analyses of a child's testimony. En F. Lösel, D. Bender y T. Bliesener (Eds.), Psychology and Law. International Perspectives (pp. 335-344). Berlin: W de Gruyter.

Bensi, L., GambetTi, E., Nori, R. y Giusberti, F. (2009). Discerning truth from deception: The sincere witness profile. The European Journal of Psychology Applied to Legal Context, 1 (1), 101-121.

Bettenay, C., Ridley, A. M., Henry, L. A. y Crane, L. (2014). Cross-examination: The testimony of children with and without intellectual disabilities. Applied Cognitive Psychology, 28 (2), 204-214. doi:10.1002/acp.2979.

Bond, C. F. y DePaulo, B. M. (2006). Accuracy of deception judgments. Personality and Social Psychology Review, 10 (3), 214-234. doi:10.1207/s15327957pspr1003_2.

Bond, G. D. (2007). Deception detection expertise. Law and Human Behavior, 32, 339-351. doi:10.1007/s10979-007-9110-z.

BotToms, B. L., NysSe-Carris, K. L., Harris, T. y Tyda, K. (2003). Juror's perceptions of adolescent sexual assault victims who have intellectual disabilities. Law and Human Behavior, 27, 205-227. doi:10.1023/A:1022551314668.

Brown, D. A. y Lewis, C. N. (2013). Competence is in the eye of the beholder: Perceptions of intellectually disabled child witnesses. International Journal of Disability, Development and Education, 60 (1), 3-17. doi:10.1080/1034912X.2013.757132.

Brown, D. A., Lewis, C. N., Lamb, M. E. y Stephens, E. (2012). The influences of delay and severity of intellectual disability on event memory in children. Journal of Consulting and Clinical Psychology, 80 (5), 829-841. doi:10.1037/a0029388.

CAMPOS, L. y AlONSO-QuECUTY, M. L. (1998). Knowledge of crime context: Improving the understanding of why the cognitive interview works. Memory, 6, 103-112. doi:10.1080/741941602.

Carlin, M., Toglia, M. P., Belmonte, C. y DiMeglio, C. (2012). Effects of presentation mode on veridical and false memory in individuals with intellectual disability. American Journal on Intellectual and Developmental Disabilities, 117 (3), 183-193. doi: 10.1352/1944-7558117.3.183.

Clarke, J., Prescott, K. y Milne, R. (2013). How effective is the cognitive interview when used with adults with intellectual disabilities specifically with conversation recall? Journal of Applied Research in Intellectual Disabilities, 26 (6), 546-556.

Collins, D. y Henry, L. (2016). Eyewitness recall and suggestibility in individuals with Down syndrome. Journal of Intellectual Disability Research, 60 (12), 1227-1231. doi:10.1111/jir.12310.

Comblain, C., D’Argembeau, A. y Van der Linden, M. (2005). Phenomenal characteristics of autobiographical memories for emotional and neutral events in older and younger adults. Experimental Aging Research, 31 (2), 173-189. doi:10.1080/03610730590915010.

Contreras, M. J., Silva, E. y Manzanero, A. L. (2015). Evaluación de capacidades para testificar en víctimas con discapacidad intelectual. Anuario de Psicología Jurídica, 25, 87-96. doi: 10.1016/j.apj.2015.02.006.

Diges, M., Rubio, M. E. y Rodríguez, M. C. (1992). Eyewitness testimony and time of day. En F. Lösel, D. Bender y T. Bliesener (Eds.), Psychology and Law. International Perspectives (pp. 317-320). Berlin: W de Gruyter. 
Ekman, P., O’Sullivan, M. y Frank, M. G. (1999). A few can catch a liar. Psychological Science, 10 (3), 263-266. doi:10.1111/1467-9280.00147.

Ericsson, K. A. y SMith, J. (1991). Toward a general theory of expertise: Prospects and limits. New York, NY: Cambridge University Press.

FACCINI, L. y SAIDE, M. A. (2011). Psychologists' experience with interviewing and analyzing abuse allegations of adults with intellectual disabilities. Sexuality and Disability, 29 (3), 291-296. doi:10.1007/s11195-011-9206-8.

Garrido, E., Masip, J. y Herrero, C. (2004). Police officers' credibility judgments: Accuracy and estimated ability. International Journal of Psychology, 39 (4), 254-275. doi: 10.1080/00207590344000411.

González, J. L., Cendra, J. y Manzanero, A. L. (2013). Prevalence of disabled people involved in Spanish Civil Guard's police activity. Research in Developmental Disabilities, 34 (11), 3781-3788. doi:10.1016/j.ridd.2013.08.003.

GonzÁlez, J. L., Muñoz, J. M., Sotoca, A. y Manzanero, A. L. (2013). Propuesta de protocolo para la conducción de la prueba preconstituida en víctimas especialmente vulnerables. Papeles del Psicólogo, 34 (3), 227-237.

Goodman, G. S., Batterman-Faunce, J. M., Schaaf, J. M. y Kenney, R. (2002). Nearly 4 years after an event: Children's eyewitness memory and adults' perceptions of children's accuracy. Child Abuse and Neglect, 26, 849-884. doi:10.1016/S0145-2134(02)00354-X.

Henkel, L. A., Franklin, N. y Johnson, M. K. (2000). Cross-modal source monitoring confusions between perceived and imagined events. Journal of Experimental Psychology: Learning, Memory and Cognition, 26 (2), 321-335. doi:10.1037/0278-7393.26.2.321.

Henry, L., Ridley, A., Perry, J. y Crane, L. (2011). Perceived credibility and eyewitness testimony of children with intellectual disabilities. Journal of Intellectual Disability Research, 55 (4), 385-391. doi:10.1111/j.1365-2788.2011.01383.x.

Johnson, M. K., Hashtroudi, S. y Lindsay, D. S. (1993). Source monitoring. Psychological Bulletin, 114, 3-28. doi:10.1037/0033-2909.114.1.3.

Johnson, M. K. y Raye, C. (1981). Reality monitoring. Psychological Review, 88, 67-85. doi: 10.1037/0033-295X.88.1.67.

Kebbell, M. R., Hatton, C. y Johnson, S. D. (2004). Witnesses with intellectual disabilities in court: What questions are asked and what influence do they have? Legal and Criminological Psychology, 9 (1), 23-35. doi:10.1348/135532504322776834.

Köhnken, G., Manzanero, A. L. y ScotT, M. T. (2015). Análisis de la Validez de las Declaraciones (SVA): mitos y limitaciones. Anuario de Psicología Jurídica, 25, 13-19. doi:10.1016/j. apj. 2015.01.004.

Landau, B. y Zukowsky, A. (2003). Objects, Motions and Paths: spatial language in children with Williams Syndrome. Developmental Neuropsychology, 23, 105-137. doi:10.1080/875 65641.2003 .9651889$.

ManN, S. y VRIJ, A. (2006). Police officers' judgements of veracity, tenseness, cognitive load and attempted behavioural control in real-life police interviews. Psychology, Crime and Law, 12 (3), 307-319. doi:10.1080/10683160600558444.

Mann, S., VRIJ, A. y Bull, R. (2004). Detecting true lies: Police officers' ability to detect suspects' lies. Journal of Applied Psychology, 89 (1), 137-149. doi:10.1037/0021-9010.89.1.137.

Manzanero, A. L. (1994). Recuerdo de sucesos complejos: Efectos de la recuperación múltiple y la tarea de recuerdo en la memoria. Anuario de Psicología Jurídica, 4, 9-23.

Manzanero, A. L. (2001). Procedimientos de evaluación de la credibilidad de las declaraciones de menores víctimas de agresiones sexuales. Revista de Psicopatología Clínica, Legal y Forense, 1 (2), 51-71. 
Manzanero, A. L. (2006). Do perceptual and suggested accounts actually differ? Psychology in Spain, 10 (1), 52-65.

Manzanero, A. L. (2009). Análisis de contenido de memorias autobiográficas falsas. Anuario de Psicología Jurídica, 19, 61-72.

Manzanero, A. L. (2010). Memoria de Testigos: Obtención y valoración de la prueba testifical. Madrid: Pirámide.

Manzanero, A. L., Alemany, A., Recio, M., Vallet, R. y Aróztegui, J. (2015). Evaluating the credibility of statements given by persons with intellectual disability. Anales de Psicología, 31 (1), 338-344. doi:10.6018/analesps.31.1.166571.

Manzanero, A. L. y Diges, M. (1994). Análisis de la credibilidad de recuerdos percibidos e imaginados. Apuntes de Psicología, 41 y 42, 81-92.

Manzanero, A. L. y Diges, M. (1995). Effects of preparation on internal and external memories. En G. Davies, S. M. A. Lloyd-Bostock, M. McMurran y C. Wilson (Eds.), Psychology, law and criminal justice. International developments in research and practice (pp. 56-63). Berlin: W. De Gruyter y Co.

Manzanero, A. L., El-Astal, S. y Aróztegui, J. (2009). Implication degree and delay on recall of events: An experimental and HDV study. The European Journal of Psychology Applied to Legal Context, 1 (2), 101-116.

Manzanero, A. L. y González, J. L. (2013). Avances en Psicología del Testimonio. Santiago de Chile: Ediciones Jurídicas de Santiago.

Manzanero, A. L. y GonzÁlez, J. L. (2015). Modelo holístico de evaluación de la prueba testifical (HELPT). Papeles del Psicólogo, 36 (2), 125-138.

Manzanero, A. L., Quintana, J. M. y Contreras, M. J. (2015). (The null) Importance of police experience on intuitive credibility of people with intellectual disabilities. Research in Developmental Disabilities, 36, 191-197. doi:10.1016/j.ridd.2014.10.009.

Manzanero, A. L., Vallet, R., Aróztegui, J. y ScotT, M. T. (en revisión). CBCA ratings in statements from true and simulated victims with intellectual disability.

Masip, J. y Herrero C. (2015). Nuevas aproximaciones en detección de mentiras I. Antecedentes y marco teórico. Papeles del Psicólogo, 36 (2), 83-95.

Masip, J., Sporer, S. L., Garrido, E. y Herrero, C. (2005). The detection of deception with the reality monitoring approach: a review of the empirical evidence. Psychology, Crime and Law, 11 (1), 99-122. doi:10.1080/10683160410001726356.

McNally, R. J., Clancy, S. A., Barrett, H. M. y Parker, H. A. (2005). Reality monitoring in adults reporting repressed, recovered, or continuous memories of childhood sexual abuse. Journal of Abnormal Psychology, 114 (1), 147-152. doi:10.1037/0021-843X.114.1.147.

Meissner, C. A. y Kassin, S. M. (2002). «He’s guilty!»: Investigator bias in judgments of truth and deception. Law and Human Behaviour, 26, 469-480. doi:10.1023/A:1020278620751.

Otgaar, H., Candel, I., Memon, A. y Almerigogna, J. (2010). Differentiating between children's true and false memories using reality monitoring criteria. Psychology, Crime $\mathcal{E}$ Law, 16 (7), 555-566. doi:10.1080/10683160902926166.

Nathanson, R. y Platt, M. D. (2005). Attorneys' perceptions of child witnesses with mental retardation. Journal of Psychiatry E Law, 33 (1), 5-42. doi:10.1177/009318530503300102.

Peled, M., Iarocci, G. y Connolly, D. A. (2004). Eyewitness testimony and perceived credibility of youth with mild intellectual disability. Journal of Intellectual Disability Research, 18 (7), 669-703. doi:10.1111/j.1365-2788.2003.00559.x.

Perlman, N. B., Ericson, K. I., Esses, V. M. e Isaacs, B. J. (1994). The developmentally handicapped witness: Competency as a function of question format. Law and Human Behaviour, 18, 171-187. doi:10.1007/BF01499014. 
Porter, S. y Yuille, J. C. (1996). The language of deceit: an investigation of the verbal cues to deception in the interrogation context. Law and Human Behavior, 20, 443-458. doi:10.1007/BF01498980.

Scott, M. T. y Manzanero, A. L. (2015). Análisis del expediente judicial: Evaluación de la validez de la prueba testifical. Papeles del Psicólogo, 36 (2), 139-144.

Sporer, S. L. (1997). The less travelled road to truth: verbal cues in deception detection in accounts of fabricated and self-experienced events. Applied Cognitive Psychology, 11, 373397. doi:10.1002/(SICI)1099-0720(199710)11:5<373::AID-ACP461>3.0.CO;2-0.

Sporer, S. L. y Sharman, S. J. (2006). Should I believe this? Reality monitoring of accounts of self-experienced and invented recent and distant autobiographical events. Applied Cognitive Psychology, 20, 837-854. doi:10.1002/acp.1234.

Steller, M. y Köhnken, G. (1989). Criteria-based statement analysis. En D. C. Raskin (Ed.), Psychological methods in criminal investigation and evidence (pp. 217-245). New York: Springer.

Stobbs, G. y KebBell, M. (2003). Juror's perception of witnesses with intellectual disabilities and influence of expert evidence. Journal of Appplied Research in Intellectual disabilities, 16, 107-114. doi:10.1046/j.1468-3148.2003.00151.x.

Strömwall, L. A., Bengtsson, L., Leander, L. y Granhag, P. A. (2004). Assessing children's statements: The impact of a repeated Experience on CBCA and RM ratings. Applied Cognitive Psychology, 18, 653-668. doi:10.1002/acp.1021.

Ternes, M. y Yuille, J. C. (2008). Eyewitness memory and eyewitness identification performance in adults with intellectual disabilities. Journal of Applied Research in Intellectual Disabilities, 21 (6), 519-531. doi:10.1111/j.1468-3148.2008.00425.x.

Undeutsch, U. (1982). Statement reality analysis. En A. TRAnkell (Ed.), Reconstructing the past (pp. 27-56). Stockholm: Norstedt and Soners.

VRIJ, A. (2005). Criteria-Based Content Analysis: A Qualitative Review of the First 37 Studies. Psychology, Public Policy, and Law, 11 (1), 3-41. doi: 10.1037/1076-8971.11.1.3.

Vrij, A., Akehurst, L., Soukara, S. y Bull, R. (2004). Detecting deceit via analysis of verbal and nonverbal behaviour in children and adults. Human Communication Research, 30 (1), 8-41. doi:10.1111/j.1468-2958.2004.tb00723.x.

VRIJ, A., Fisher, R., Mann, S. y Leal, S. (2006). Detecting deception by manipulating cognitive load. Trends in Cognitive Sciences, 10, 141-142. doi:10.1016/j.tics.2006.02.003.

VRIJ, A. y Graham, S. (1997). Individual differences between liars and the ability to detect lies. Expert Evidence, 5, 144-148. doi:10.1023/A:1008835204584.

VRIJ, A. y Heaven, S. (1999). Vocal and verbal indicators of deception as a function of lie complexity. Psychology, Crime and Law, 5, 203-215. doi:10.1080/10683169908401767. 